\title{
Parallel-kinematics XYZ MEMS Part 2: Fabrication and Experimental Characterization
}

\author{
Bonjin Koo, Jorge Correa and Placid Ferreira* \\ Department of Mechanical Science and Engineering, 1206 West Green Street, MC-244, University of Illinois at Urbana-Champaign, Urbana, IL 61801, USA
}

\begin{abstract}
This paper, the second of a set of two papers addressing parallel-kinematics MEMS stages for spatial translation, deals with fabrication, characterization and control of such devices. Double device layer SOI (Silicon-On-Insulator) substrates are used, providing three layers (two device layers and the handle) into which the elements of the stage can be mapped. Using the mechanism concept, realization scheme, and kinematic and dynamic models developed in the first paper of this set, this paper provides a detailed approach to fabricating these devices. The stages fabricated have a workspace cube of roughly 20 microns on the side, an in-plane stiffness of $96 \mathrm{~N} / \mathrm{m}$, and an out-of-plane stiffness of 166 $\mathrm{N} / \mathrm{m}$. Further, it characterizes the performance of the individual actuating and sensing elements, configures feedback controllers for each actuated joint, and assesses and verifies the stage's designed performance. Finally, it demonstrates full 3-axis, closed-loop positioning of a MEMS stage.
\end{abstract}

Index Terms - XYZ MEMS, parallel-kinematics mechanisms, nanopositioning,

\section{Introduction}

Tn the companion paper [1], we have discussed the kinematics, dynamics and design approach to a parallel-kinematics, MEMSscale, spatial translation stage concept (referred to as a PK-XYZ-MEMS stage), realizable on a double device layer silicon-oninsulator (SOI) substrate. We have shown that the forward position kinematics problem is reduced to a sixth-order polynomial equation, and that the inverse position kinematics problem admits closed-form solution. The expressions for Jacobian matrices relating the end-effector motion to displacements at flexure joints, near to the nominal configuration, were derived, Further, formulae for the compliance of the critical components of the stage, leading to the construction of the theoretical workspace and an analytic characterization of the statics and dynamics of the stage.

The purpose of this paper is to discuss the physical realization of a PK-XYZ-MEMS stage, explain the fabrication process, experimentally characterize its workspace, verify its mobility, and validate its static and dynamic performance. The companion paper [1] also reported on the design methodology of a PK-XYZ-MEMS stage and, while elucidating the design methodology and verifying the kinematic and dynamic equations, produced a detailed design of a MEMS stage with previously unrealized mechanical specifications. The reader may find it useful to refer back to [1] for details of the formulae and procedures used. Section II of this paper reviews the design objectives, motivation, and design rationale and summarizes aspects of the design that are important from the point of view of fabrication. It adds information on the sensing combs, necessary for configuring the closed loop controllers for the stage's actuators. Section III details the fabrication steps and describes the fabrication results. Section IV characterizes the individual actuators and sensors in each kinematic chain of the mechanism, using this information to configure closed-loop controllers for each actuator. Section V tests the kinematics, statics and dynamics of the stage. Finally, Section VI summarizes the results and draws conclusions.

\section{Device Architecture}

A schematic of the PK-XYZ-MEMS mechanism with key design parameters and variables is given in Figure 1 (a). Figure 1(b) gives a CAD rendering of the designed stage, showing the details of its layout in a double device layer SOI substrate. Figure 1(c) shows a single kinematic chain in the mechanism with key design parameters of the flexure joints in the chain identified. The designed device is expected to be a general-purpose, MEMS-scale, spatial positioning platform that can be adapted to several possible applications. Motivated by applications in nano-indentation, confocal microscopy, scanned probe microscopy, the design objectives are to realize an in-substrate, low-profile stage based on the kinematic scheme developed in [1], and actuated by electrostatic drives. The stage is designed to have workspace capable of embedding a cube of dimension $20 \mu \mathrm{m} \times 20 \mu \mathrm{m} \times 20 \mu \mathrm{m}$ and is to be actuated by electrostatic drives with a maximum voltage of less than $150 \mathrm{~V}$. It is designed to have a positioning resolution better than $10 \mathrm{~nm}$, with stiffness better than $75 \mathrm{~N} / \mathrm{m}$ and a natural frequency around 500-1000 Hz.

The fabrication of the PK-XYZ-MEMS stage represents a challenging task because of the 3D features needed by its kinematic structure and the inherently $2-2.5 \mathrm{D}$ nature of the common fabrication processes. To address this issue, the 3D structure of the manipulator is mapped into three layers: the device layers and the handle at different Z-heights in the substrate. As depicted in Figure 1(b), and explained in [1], in this architecture, the 4-bar mechanism, the comb drives and the first set of out-of-plane joints are mapped onto the device layer 1(DL1). The end-effector and the remaining set of out of plane hinges are mapped to the device 
layer 2 (DL2) and, finally, the out-of-plane links are patterned across the handle and the BOX layers to connect the structures in the two layers. The use of the double device layer substrate, while not providing the same level of flexibility in realizable geometries as that provided by processes such as polyMUMPS [2], overcomes the complexity of depositing and patterning multiple layers of polysilicon and oxides. Most importantly, it overcomes the limitations of layer thickness and provides more options for realizing different out-of-plane dimensions. This is particularly important in designing and realizing mechanisms that must provide out-ofplane movement as it obviates the need for very large actuation forces to move the mechanism out from a singular, initial planar configuration. With a metal layer on one of the device layers, the use of a double device layer SOI substrate provides six layers, with the BOX layers available to play the role of etch stop and sacrificial layers during fabrication, and electrical insulation layers during functioning of the device.

In previous work [3 - 6], our group has demonstrated the fabrication of single-axis and 2 degree-of-freedom translational MEMS stages on SOI wafer substrates. This was convenient due to the fact that the actuation layer, the kinematic structure, and endeffector were all patterned together on the device layer of the SOI, while the handle and the BOX layers where selectively etched to produce a free standing structure. In the case of the PK-XYZ-MEMS stage, the connection of the patterns in the two device layers with a link embedded in the handle requires the fabrication of the out-of-plane hinges. These hinges are typically realized by selectively reducing the cross-section of the substrate through a non-self-limiting or timed etching process. This can be challenging to control, especially when etching deep into the wafer and requiring hinge thicknesses less than 10 microns. The use of a double device layer SOI wafer as the primary substrate, provides an extra BOX layer and hence an etch stop, thus avoiding the need of precise depth control while etching from the two opposite faces of the wafer to generate out-of-plane hinges at different depths in the substrate that join the patterns on the two faces of the substrate. This greatly reduces the complexity of the fabrication process.

The critical considerations in the selection of the wafer are the thicknesses of the handle and the device layers. An "oreo" wafer Ultrasil D4-6110 with device layer thicknesses of $90 \mu \mathrm{m}$ and $27 \mu \mathrm{m}$, handle layer thickness of $300 \mu \mathrm{m}$, and BOX layers' thickness of $0.5 \mu \mathrm{m}$ was selected. The $300 \mu \mathrm{m}$ handle thickness was large enough to produce an initial elevation angle $\alpha_{\mathrm{o}}$ of $31.9^{\circ}$ and reduce the actuation forces required of the combs (note that $0^{\circ}$ would be a singularity, theoretically requiring infinitely large forces to cause motion) to an acceptable level while, at the same time, reducing the etch depth to a manageable level. This elevation angle corresponds to mechanical advantage close to 0.5 . The asymmetry in the device layer thicknesses is necessitated by need to limit twisting and out-of-plane deflection in the folded leaf-springs in actuation layer due to out-of-plane forces.

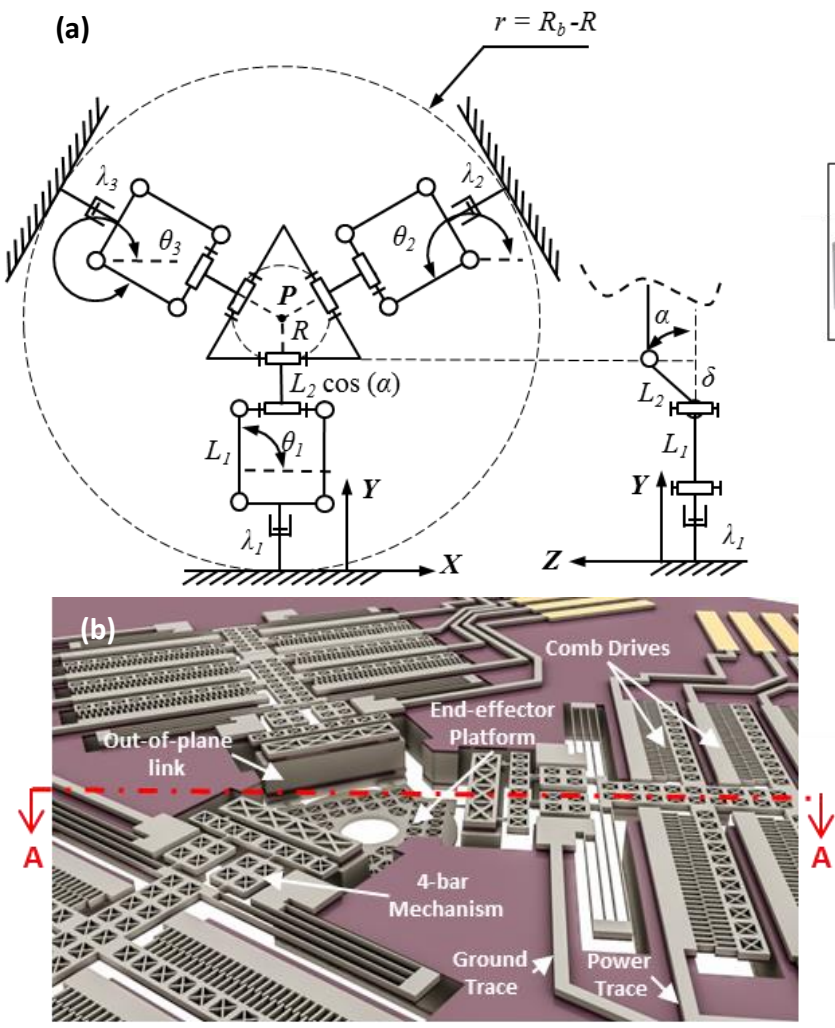

Section AA

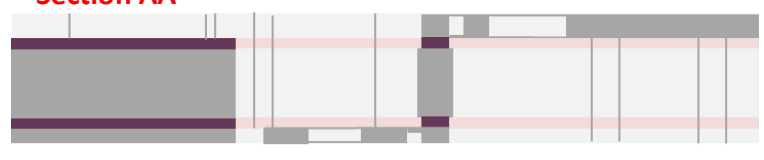

4-bar mechanism

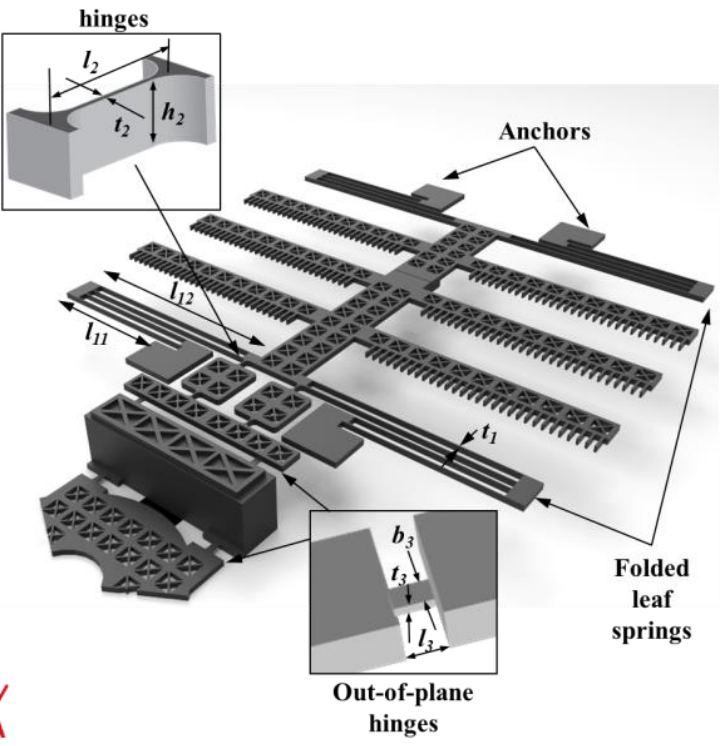

(c)

Figure 1. (a) Schematic of PK-XYZ-MEMS kinematics showing the important global parameters and variables. (b) 3$\mathrm{D}$ rendering of stage layout showing important elements. DL1 Different layers (Device Layers 1 and 2, Handle, and BOX BOX layers) of the substrate are visible in section. (c) A solid model HL of a kinematic chain with flexure joints and important BOX parameters defining them. 
Table 1. Design parameters for the PK-XYZ-MEMS stage

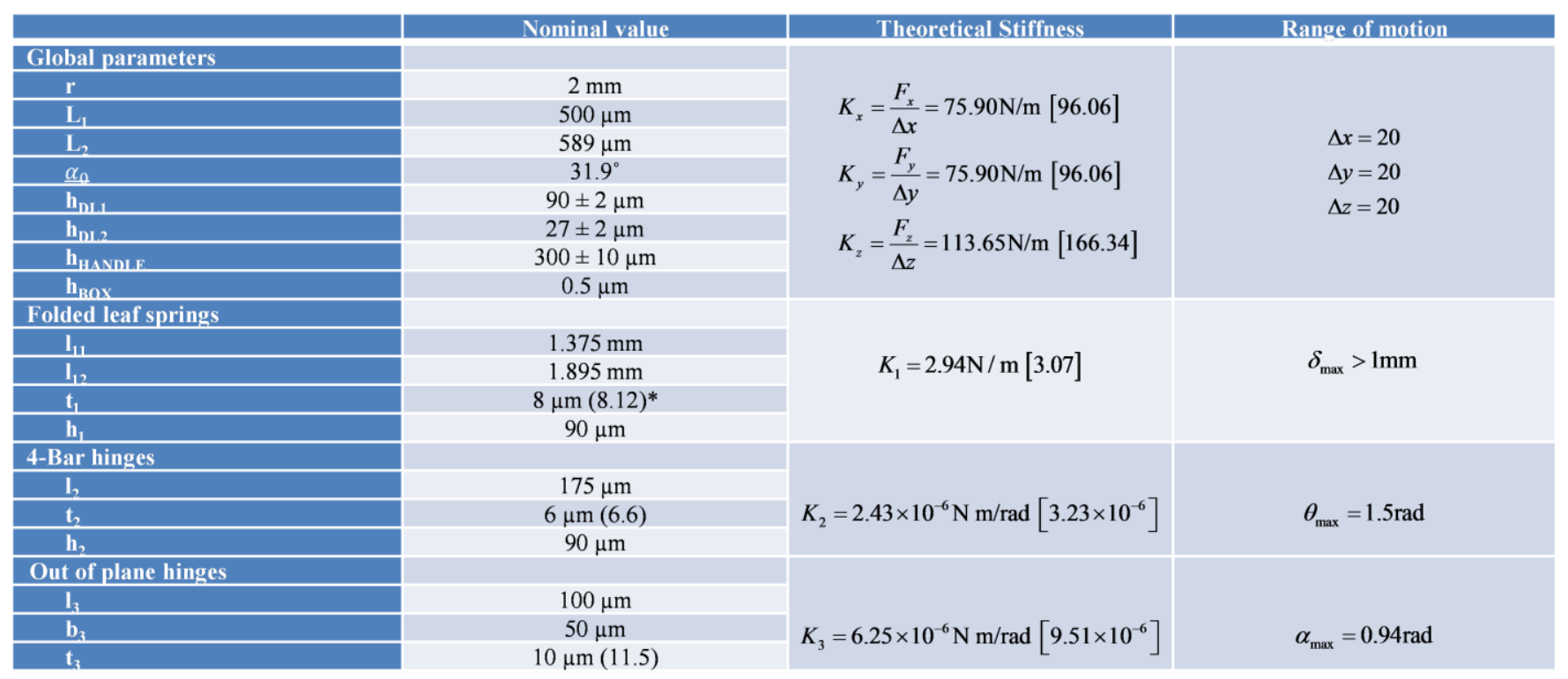

\footnotetext{
*Values in parenthesis () are values measured after fabrication

**Values in brackets [] are the predicted based on measured dimensions.
}

In previous work [3], [7], [8], we have designed, developed and tuned fabrication processes and characterized several elements such as comb drives, folded leaf-springs, flexure hinges, capacitive sensors for positioning systems. Many of these designs are adapted for use in this device. Using the motivation given above and the design methodology given in [1], the kinematic parameters (see Figure 1(a-c)), the dimensions of flexible elements and theoretical performance of the different design elements used in the mechanism are summarized in Table 1.

A summary of the design layout of the device along with a discussion of the design intent used for the critical components in the mechanism is given below.

\section{A. Top Device Layer (DL1)}

The top device layer of the "oreo" wafer carries the electrostatic comb drives, the folded spring suspensions and the four-bar mechanisms and the upper out-of-plane hinges. Each of the three kinematic chains of the manipulator is equipped with two sets of electrostatic combs, one for actuation and the other for sensing. Together, they become the closed-loop controlled prismatic actuator for each chain. The actuation combs are designed with 360 fingers with $90 \mu \mathrm{m}$ finger height (DL1 thickness) and $5 \mu \mathrm{m}$ gaps. The gap is determined by our process resolution limits, while the 360 fingers produce a desired maximum force of $1 \mathrm{mN}$ at $130 \mathrm{~V}$.

The sensing combs are designed to work with a commercial-off-the-shelf (COTS) capacitance-to-voltage conversion chip, the MS 3110 whose output is digitized by a 16-bit analog-to-digital converter (ADC) and produce a sensing resolution of $1 \mathrm{~nm}$ (see [3] for design procedure). Thus the sensing comb is designed as a differential comb with 360 fingers that produces a capacitance change of $0.0574 \mathrm{pF}$ per micron of displacement.

Patterned in DL1, the moving elements of actuation and sensing combs are suspended on the same set of folded-leaf springs and are mechanically connected. However, they need to be electrically isolated to prevent the high voltage signal from the actuation circuit from leaking to the low voltage sensing circuit while maintaining the physical connection of the structure. To meet this requirement, a channel is patterned across the device layer (DL1) to provide the electrical isolation and lap joints are patterned across the BOX and handle layers to ensure mechanical attachment [3].

The function of the folded leaf-springs (see Table 1) is to anchor the suspended combs and the kinematic chain to the substrate while providing it a translational degree of freedom. Together with the comb drive, it realizes the actuated prismatic joint of the kinematic chain. The folded leaf-spring design allows for an increased range of motion while providing high lateral and out-ofplane stiffness.

A parallelogram 4-bar mechanism with flexure hinges (parameters in Table 1), located between the anchors of the folded leafsprings with its base connected to the moving combs of the actuators, provides a free lateral (in-plane, perpendicular to the motion of the comb) degree of freedom The connecting link carries the out-of-plane link (patterned in the handle layer) and attach to it by a pair of out-of-plane hinges (see Table 1 and Figure 1) that define an in-plane axis of rotation. 


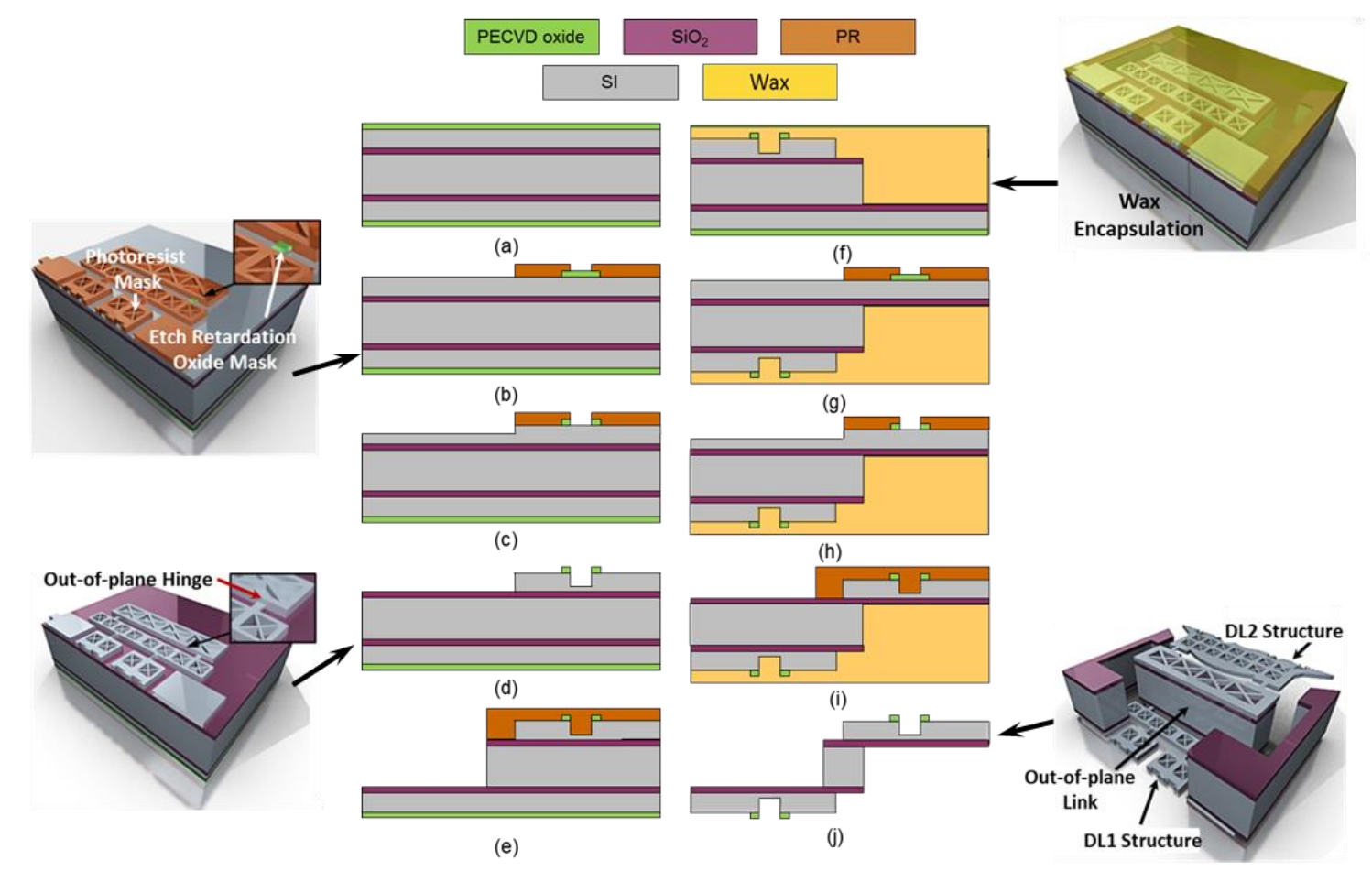

Figure 2. Fabrication steps to produce the PK-XYZ-MEMS stage

\section{B. Bottom Device Layer (DL2)}

The end-effector and the second set of out-of-plane hinges are located in bottom device layer (DL2). The end-effector has a circular shape patterned with an inner circular cavity for the modular assembly of additional features for possible optical and mechanical applications. A truss-like structure pattern is used to reduce the mass of the end-effector (and also of all in-plane, moving links of the mechanism), while maintaining structural integrity. Six out-of-plane hinges connect the out-of-plain links of the 3 kinematic chains to the end effector allowing for the vertical motion and preventing the parasitic rotations.

\section{Handle and BOX layers (HL and BOX)}

The handle layer has two critical components, namely, the out-of-plane links and the lap joints. As mentioned before, the lap joints mechanically attach the sensing and the actuation combs while electrically isolating them. The out-of-plane links are patterned across the BOX and the handle layers and they connect the two device layers (DL1 and DL2) involved in the fabrication process to allow the vertical motion of the manipulator. These links are designed with hollow box sections to reduce mass.

The BOX layer is mainly used to provide an etch stop when etching through the handle, DL1 and DL2, as well as to electrically isolate the actuation and the sensing comb drives.

\section{Fabrication Process}

The overall fabrication process for the PK-XYZ-MEMS requires only the standard use of conventional lithography-based microfabrication processes because of the use of the double device layer SOI substrate and the careful mapping and layout of the mechanism's elements in the layers provided by it.

The stage is fabricated on a $17 \mathrm{~mm}$ x $17 \mathrm{~mm}$ substrate diced out from the "oreo" wafer and cleaned with a RC-1 standard cleaning procedure. The fabrication process is summarized in Figure 2. In Figure 2(a), a 150nm layer of PECVD oxide is deposited on both top (DL1) and bottom device layers (DL2) using the Plasmalab PECVD (Plasmalab system 100) system. The resultant oxide layer is patterned using conventional photolithography to form a temporary local etch retardation masks for realizing outof-plane hinges. Next, a photoresist mask containing features (such as the comb drives, the folded springs and 4-bar mechanisms, etc.) that reside in DL1 is patterned. It is important that the photoresist mask and the temporary PECVD oxide masks overlap to ensure proper connectivity of the out-of-plane hinges with the rest of structural elements in DL1. Figure 2(b) shows the result of this operation. A two-step DRIE process with an intermediate RIE step is carried out to finalize the fabrication of the top device layer (DL1). The first DRIE step etches DL1 to a depth equal to the desired thickness of the out-of-plane hinge, in this case $10 \mu \mathrm{m}$. This is followed by a RIE step to remove the exposed patterned retardation PECVD oxide layer (Figure 2(c)). The second DRIE operation completes the etching of DL1, encountering and exposing the BOX layer (Figure 2(d)). At this point, the patterning of DL1 is completed and the handle layer must be etched to produce features necessary for the out-of-plane link. For this purpose, a new photoresist mask is patterned on top of DL1 and followed by a HF etch of the buried oxide. The photoresist layer serves as a mask for the DRIE excavation of the handle layer as well as a protection film for the structures in the top device layer (DL1). Here 

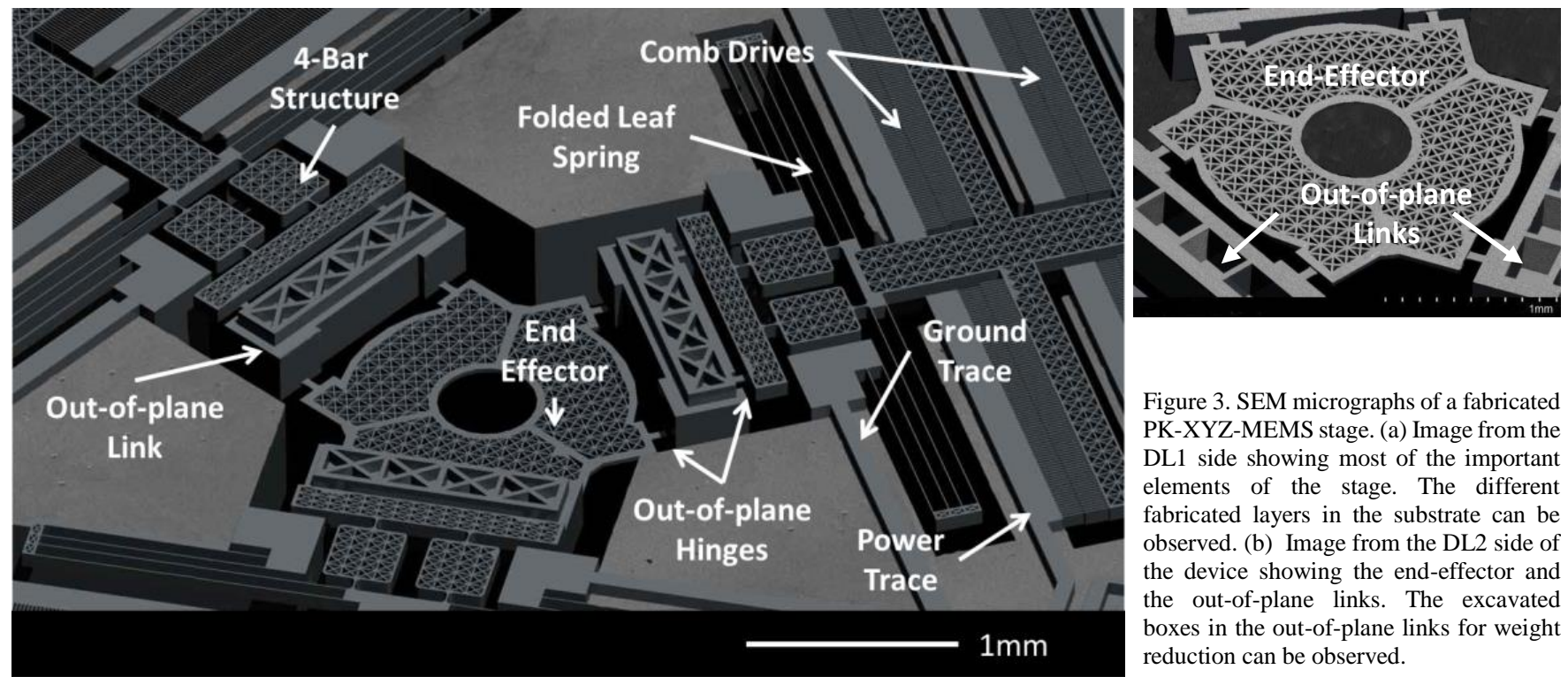

Figure 3. SEM micrographs of a fabricated PK-XYZ-MEMS stage. (a) Image from the DL1 side showing most of the important elements of the stage. The different fabricated layers in the substrate can be observed. (b) Image from the DL2 side of the device showing the end-effector and the out-of-plane links. The excavated boxes in the out-of-plane links for weight reduction can be observed.

the BOX layer between the handle and DL2 acts as a convenient etch stop. Figure 2(e) shows the cross-section view of the device after the DRIE operation to the handle.

Before proceeding with the fabrication from the other side of the substrate, i.e., the bottom device layer (DL2), a water soluble thermal wax (Crystalbond 555 Mounting Adhesive) is used to fill the cavity on top of the end- effector and encapsulate the structures in DL1. This step is performed to provide a heat sink for subsequent DRIE operations on DL2 and the handle that would otherwise produce high temperature gradients across the device layer, resulting in an uneven etch rate and the potential to break weak components such as the hinges. The same process described for DL1 is repeated for DL2, this time with sets of masks to pattern the end-effector and out-of-plane hinges in DL2 and complete the evacuation of the handle layer to leave behind the outof-plane links connecting the patterns in DL1 and DL2. Figure 2(f)-(j) depicts these repeated steps for the fabrication of the endeffector, the lower set of out-of-plane hinges and the completion/release of the out-of-plane links in the handle. Following the removal of the wax, undercut etching of the BOX layers, produces the suspended structures in DL1 and DL2. Figure 3 shows an image of the results of this fabrication process. Six SEM frames are stitched together to produce a large-area image. The critical flexure dimensions are measured and reported in Table 1. Based on these measurements, predicted values for stiffness of the elements and the stage are computed.

\section{Characterization and Calibration}

The verification of the ability of the stage to generate controllable spatial translation, consistent with the predictions made by the kinematic and dynamic models presented in the first of this set of papers [1] is addressed in this section. Since the motion is spatial and generated by the combined effect of three actuators in interacting kinematic chains, this can be challenging. Simultaneous measurement of all three spatial coordinates of the platform is difficult; therefore we verify its out-of-plane motion (along the zaxis) separately from its in-plane motion. Controlling the motion at the platform or end-effector of the stage, requires the simultaneous control of all three actuators. Further, the actuators produce internal loads on each other. Therefore, we first characterize the actuator and sensor for each kinematic chain with the other two dormant, and use this information to construct

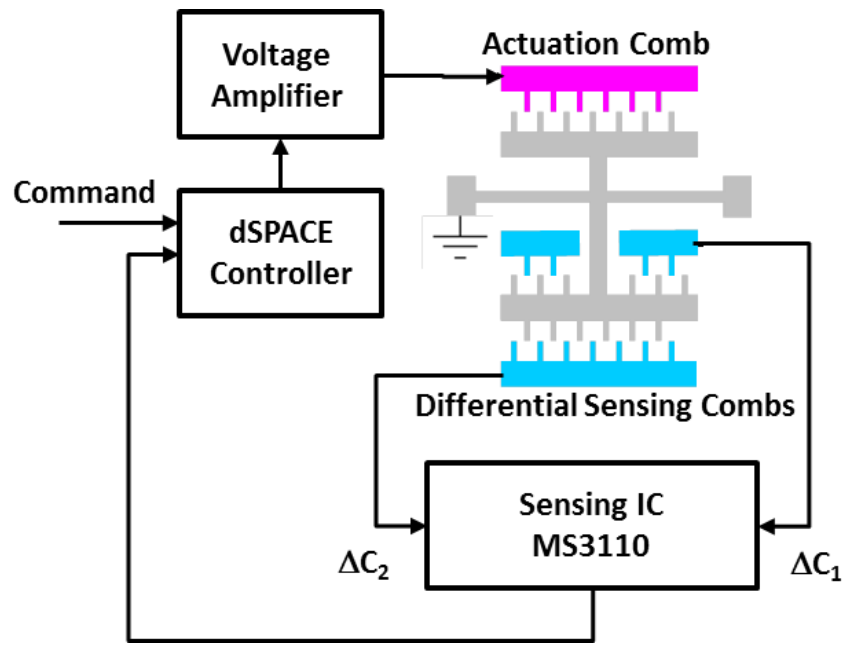

Figure 4. Circuit diagram for the actuation and sensing needed for control implementation 

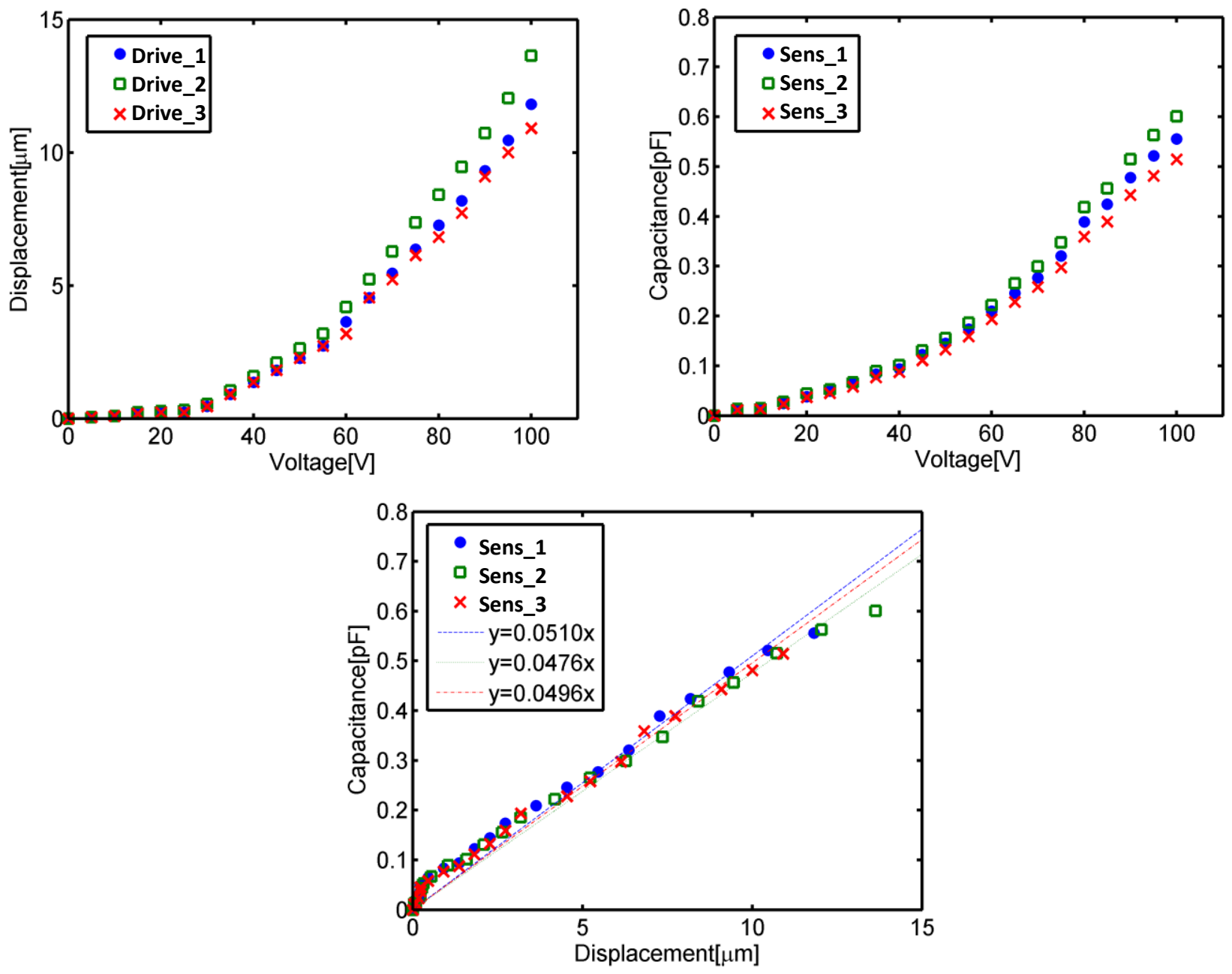

Figure 5. (a) Actuation comb drive displacement vs. actuation voltage (b) Sensing comb capacitance vs. actuation voltage (c) Actuation comb displacement vs. sensing comb capacitance

feedback controllers to regulate their positions, attenuating their response to force disturbances that they may experience as a result of their mutual interactions. With the ability to control the position of each comb drive under varying loads, the kinematics of the stage can then be verified by programming in the positions of the comb drives and observing the motion of the end-effector. The circuit schematic shown in Figure 4 is used for these calibrations. To generate the actuation voltage signal, a CEDRAT LA 75 amplifier with maximum output voltage of 150 volts was used. The capacitance change due to displacement of the sensing combs is measured with a commercial-off-the-shelf (COTS) capacitance-to-voltage conversion IC, the MS3110 chip. To reduce measurement noise, differential capacitance measurements are made and the sensing combs are electrically isolated from the rest of the stage and substrate. The substrate is directly mounted on the measurement IC board that is powered by a battery cell to isolate it from noise in the power lines [3]. A dSPACE control board implements the controller for the closed loop system.

\section{A. Characterization of actuating and sensing combs}

The voltage-displacement characteristics of actuation combs are obtained along with the displacement-capacitance characteristics of the sensing combs. This data is used to construct rudimentary closed-loop controllers for the actuators in each kinematic chain of the stage. The closed loop control compensates for internal forces in the system and possible variations in stiffness of the mechanism to permit verification of its kinematics. The actuation comb is driven by a Keithley 237 low-noise voltage source. With voltage increasing in steps of $5 \mathrm{~V}$ in the range of $0-100 \mathrm{~V}$, the displacement of the comb fingers is recorded with a 1-micrometer resolution microscope. Simultaneously, the capacitance of the sensing combs is recorded using the MS3110 capacitance measurement IC. This is repeated for the actuation and sensing combs in each of the kinematic chains. The voltagedisplacement and voltage-capacitance characteristics of the actuation and sensing combs, measured by this procedure are shown in Figure 5(a) and 5(b), respectively. Further, displacement-capacitance characteristics of the sensing combs, shown in Figure 5(c) are derived by taking displacement from Figure 5(a) and the capacitance from Figure 5(b). A linear approximation over this data yield sensitivities of $0.0510 \mathrm{pF} / \mu \mathrm{m}, 0.0476 \mathrm{pF} / \mu \mathrm{m}$, and $0.0496 \mathrm{pF} / \mu \mathrm{m}$ for the sensing combs in the first, the second and the third 


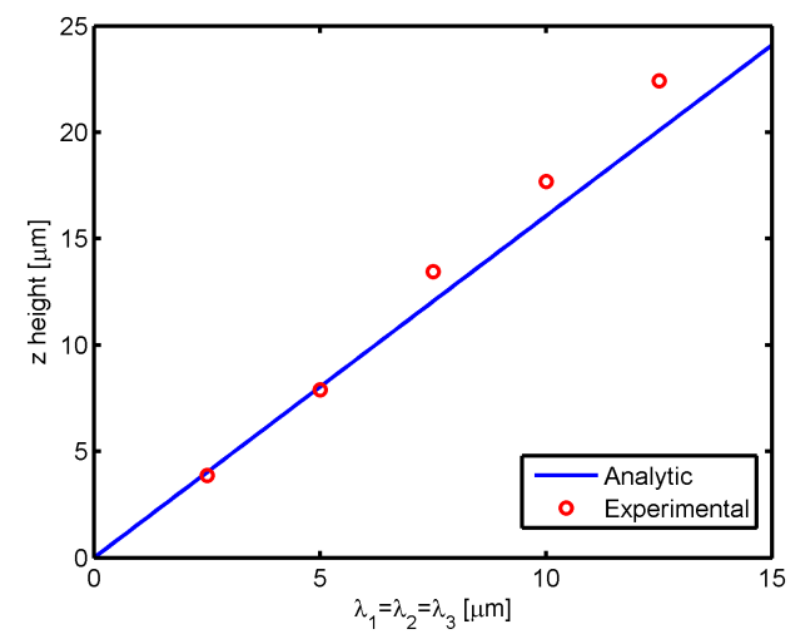

Figure 6. End effector height while comb drive position changes

kinematic chains, respectively. These values are less than the designed value of $0.0574 \mathrm{pF} / \mu \mathrm{m}$, possibly due to over etching of the comb fingers during the DRIE process.

Based on the aforementioned results, a proportional-integral (PI) controller is implemented using the technique outlined in [3, 6]. The closed-loop step and frequency response of the system are obtained to verify the performance of the PI controller. This information is presented in Appendix A.

\section{B. Characterization of Stage Kinematics}

We characterize the stage kinematics by verifying its out-of-plane and in-plane motion capabilities. As reported in our discussion of the PK-XYZ-MEMS kinematics [1], because of its in-plane symmetry, equal displacements at the three actuators produce a vertical displacement of the end-effector. The aforementioned closed-loop controllers for the actuators makes it possible for them to faithfully generate any commanded displacement as it makes them insensitive to internal loads that are generated when they are simultaneously actuated. The out-of-plane motion of the end-effector platform is measured using an active probe sensor [6], developed in our laboratory, and capable of measuring displacements and forces with resolutions of $5 \mathrm{~nm}$ and $25 \mathrm{nN}$, respectively. Among the reasons why such a specialized device is used, is the fact that the end effector of the XYZ stage was patterned with a circular hole, for future optical applications, and a truss structure to reduce mass and enhance its dynamic performance. This resulted in a mesh type surface hard to target with a capacitive or an interferometer-based displacement sensor. Figure 6 shows the analytic solution (from the linearized kinematics of the machine) and the experimental results of out-of-plane motion calibration. The discrepancy grows larger as the machine moves vertically away from its nominal configuration. This is due to the dependence of the out-of-plane motion on the elevation angle $\alpha$ of the out-of-plane link [1].

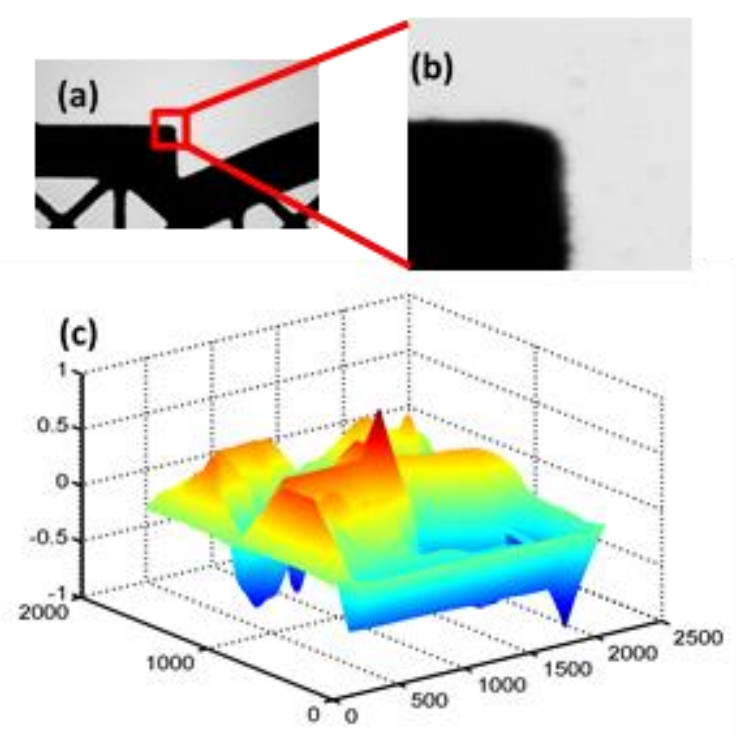

Figure 7. (a) Reference image of end-effector (b) Selected kernel (c) Surface plot of cross correlation coefficient 
To validate the in-plane kinematics and, in general, the workspace of the mechanism, the end-effector of the manipulator was sequentially commanded to move to a set of equally spaced points on the circumference of circles in XY planes located at different heights in the stage's workspace. This test was carried for the heights of $10 \mu \mathrm{m}, 15 \mu \mathrm{m}$, and $20 \mu \mathrm{m}$, related to the cross sections of
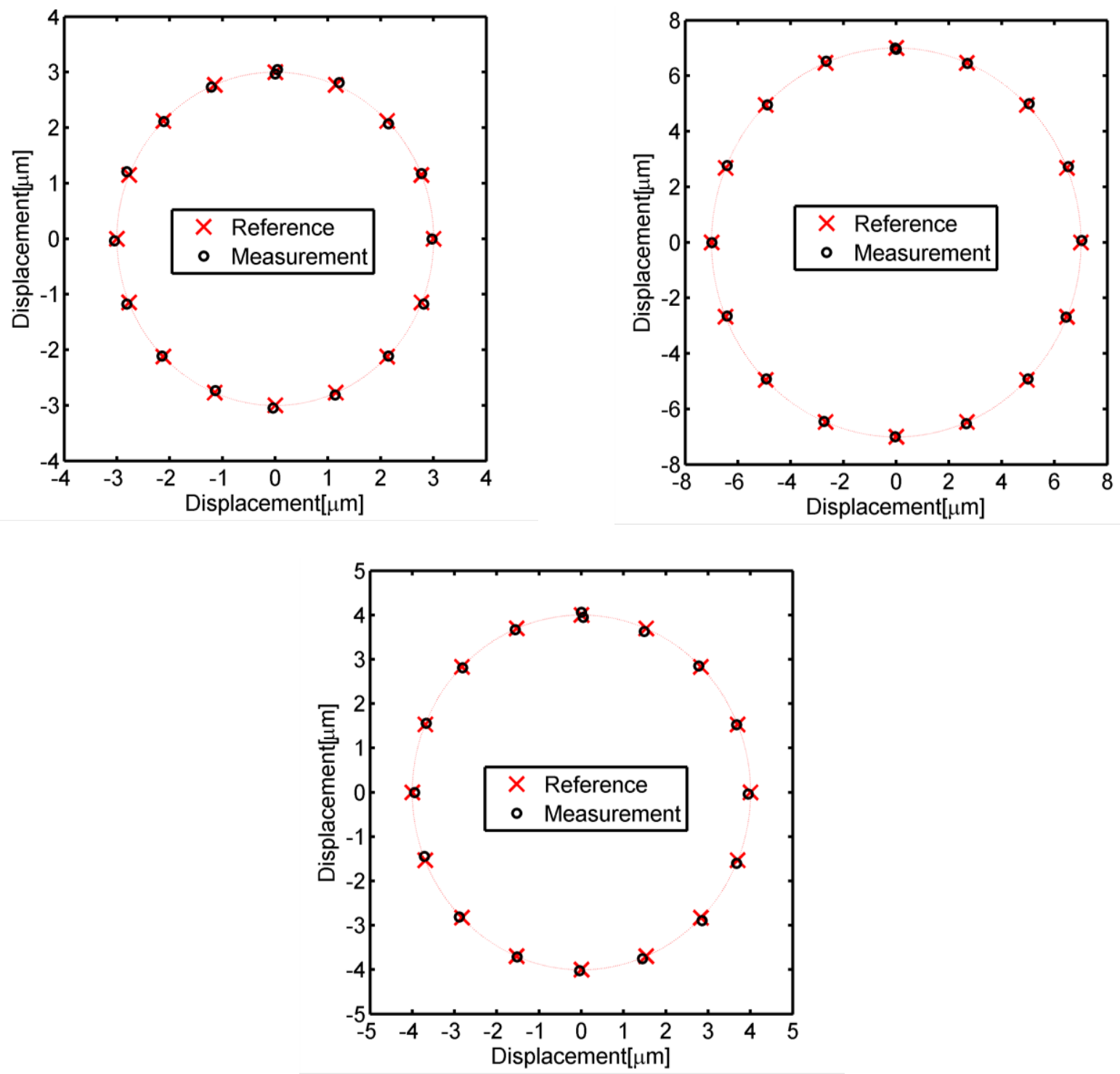

Figure 8. Circular trajectory for different end-effector heights

the theoretical workspace shown in Figure 6 of Part I of this set of papers [1]. At these heights, 16 equally space points (i.e., at angular increments of $22.5^{\circ}$ ) on circles with diameters of $6 \mu \mathrm{m} 14 \mu \mathrm{m}$, and $8 \mu \mathrm{m}$ are chosen as target points for the end-effector. The inverse kinematic equations [1] are used to calculate the actuator displacements for moving the end-effectors to each of these locations as well as to the centers of each of the circles. These actuator positions are then used as reference input for the closedloop controller. The motion of end-effector is captured with a stationary camera in high resolution microscope (Olympus MX50TF) with a pixel-to-displacement ratio resolution of $39 \mathrm{~nm} /$ pixel. A cross correlation imaging processing procedure [9] is used to compute the actual displacement (relative to the frame that corresponds to the location of the end-effector when positioned at the center of the circle. Figure 7(a) shows an image frame stored for a particular position. Within this image frame, the displacement is calculated based on the location of a 280x280 pixel kernel shown in Figure 7(b) and selected to be sensitive to both $\mathrm{x}$ and y displacements. The $\mathrm{x}$ and $\mathrm{y}$ displacements of a frame is calculated as those $\mathrm{x}$ and $\mathrm{y}$ translations that maximize the pixel-to-pixel correlation in the chosen kernel with reference frame. The method is limited by the pixel-to-displacement resolution and errors due to fluctuations in lighting and small vibrations in the camera relative to the stage.

Figure 8 shows the three circles with the reference/commanded positions and the measured locations of the end-effector. The statistics of these measurements are listed in Table 2. Given that the best resolution of the measurement system is about $40 \mathrm{~nm}$ and, with errors, is probably in the neighborhood of 50 or $60 \mathrm{~nm}$, one can conclude that the actual stage kinematics are very close to the theoretically designed kinematics, and the static errors are within the specified tolerances. 
Table 2. Statistics of measured errors in position of the end-effector for three circles in three different XY plans at different heights.

\begin{tabular}{|c|c|c|c|}
\hline $\begin{array}{c}\text { Diameter } \\
{[\mu \mathrm{m}]}\end{array}$ & $\begin{array}{c}\mathrm{Z} \\
{[\mu \mathrm{m}]}\end{array}$ & $\begin{array}{c}\text { MEAN } \\
{[\mathrm{nm}]}\end{array}$ & $\begin{array}{c}\text { STD } \\
{[\mathrm{nm}]}\end{array}$ \\
\hline 6 & 10 & 47.3 & 18.6 \\
\hline 14 & 15 & 57.2 & 23.1 \\
\hline 8 & 20 & 57.5 & 27.1 \\
\hline
\end{tabular}

\section{Static Force Capabilities in Z Direction}

The stiffness of the stage in the $\mathrm{Z}$ direction, $K_{z}$ is defined as the magnitude of force acting in the $\mathrm{Z}$ direction on the end effector required to cause it to displace by 1 length unit in the same direction. From [1], the analytic formula for the stiffness in the Zdirection is given as:

$$
K_{z}=\left[4 K_{3} \sec [\alpha]^{2} / L_{2}^{2}+12 K_{1} \tan [\alpha]^{2}\right]
$$

where $K_{3}$ is the stiffness of the out-of-plane hinge, $K_{l}$ is the stiffness of the folded spring, $L_{2}$ is the length of the out-of-plane link and $\alpha$ is the angle it makes with the XY plane. Using the measured values for the flexible elements in Table 1 to compute $K_{3}$ and $K_{1}$, the predicted stiffness in the vertical direction, $K_{z}$, becomes $166.34 \mathrm{~N} / \mathrm{m}$.

To determine an experimental value for this parameter, we used an energy based methodology [6] in which the total electrical potential energy stored in the system as a capacitance change in comb actuators equals the mechanical strain energy stored in the hinges and springs as the end-effector is displaced. Here, the resultant value of $K_{z}$ amounts for the combined resistance of individual

Table 3. LCR meter reading and motion in z-direction

\begin{tabular}{|c|c|c|c|c|}
\hline $\begin{array}{c}\mathrm{V} \\
{[\mathrm{V}]}\end{array}$ & $\begin{array}{c}\Delta \mathrm{C} \\
{[\mathrm{pF}]}\end{array}$ & $\begin{array}{c}\Delta \mathrm{E} \\
{[\mathrm{pJ}]}\end{array}$ & $\begin{array}{c}\mathrm{Z} \\
{[\mathrm{um}]}\end{array}$ & $\begin{array}{c}\mathrm{K}_{\mathrm{z}} \\
{[\mathrm{N} / \mathrm{m}]}\end{array}$ \\
\hline 20 & $8.403 \mathrm{E}-02$ & 16.81 & 0.452 & 164.52 \\
\hline 30 & $1.769 \mathrm{E}-01$ & 79.59 & 1.017 & 153.91 \\
\hline 40 & $3.137 \mathrm{E}-01$ & 250.9 & 1.808 & 153.54 \\
\hline
\end{tabular}

joints of the stage to generate motion of the end-effector in the Z-direction. From the energy balance:

$$
\frac{1}{2} \Delta C V^{2}=\frac{1}{2} K_{z} \Delta Z^{2} \text { or } K_{z}=\frac{\Delta C V^{2}}{\Delta Z^{2}}
$$

Where $\Delta C$ is the total change in capacitance in the actuation combs when a $V$ is the voltage is simultaneously applied to all of them, and $\Delta Z$ is the total displacement of the end-effector platform in the Z-direction.

The three actuation combs are connected in parallel and driven by the same voltage from an Agilent 4284A LCR-meter, which simultaneously measures the total change in capacitance at these combs. Since actuation comb drives are connected in parallel, the measured capacitance is the sum of the individual changes. Figure 9 shows the total change in capacitance at the actuation combs

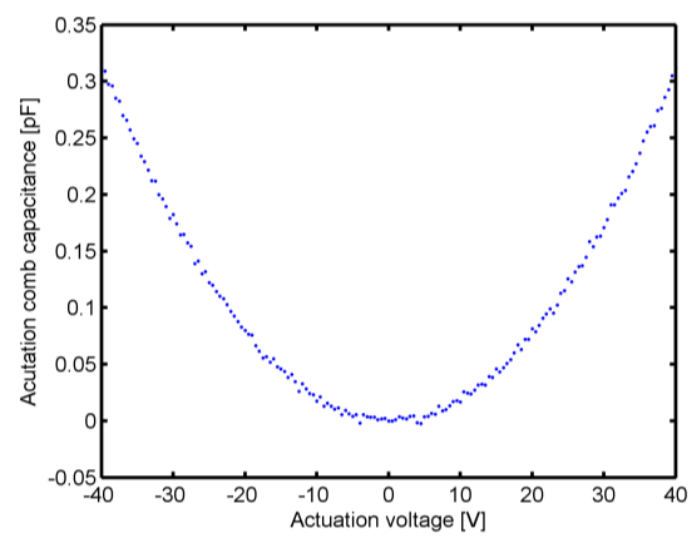

Figure 9. Capacitance vs. actuation voltage for actuation comb drive as read by an LCR-meter 

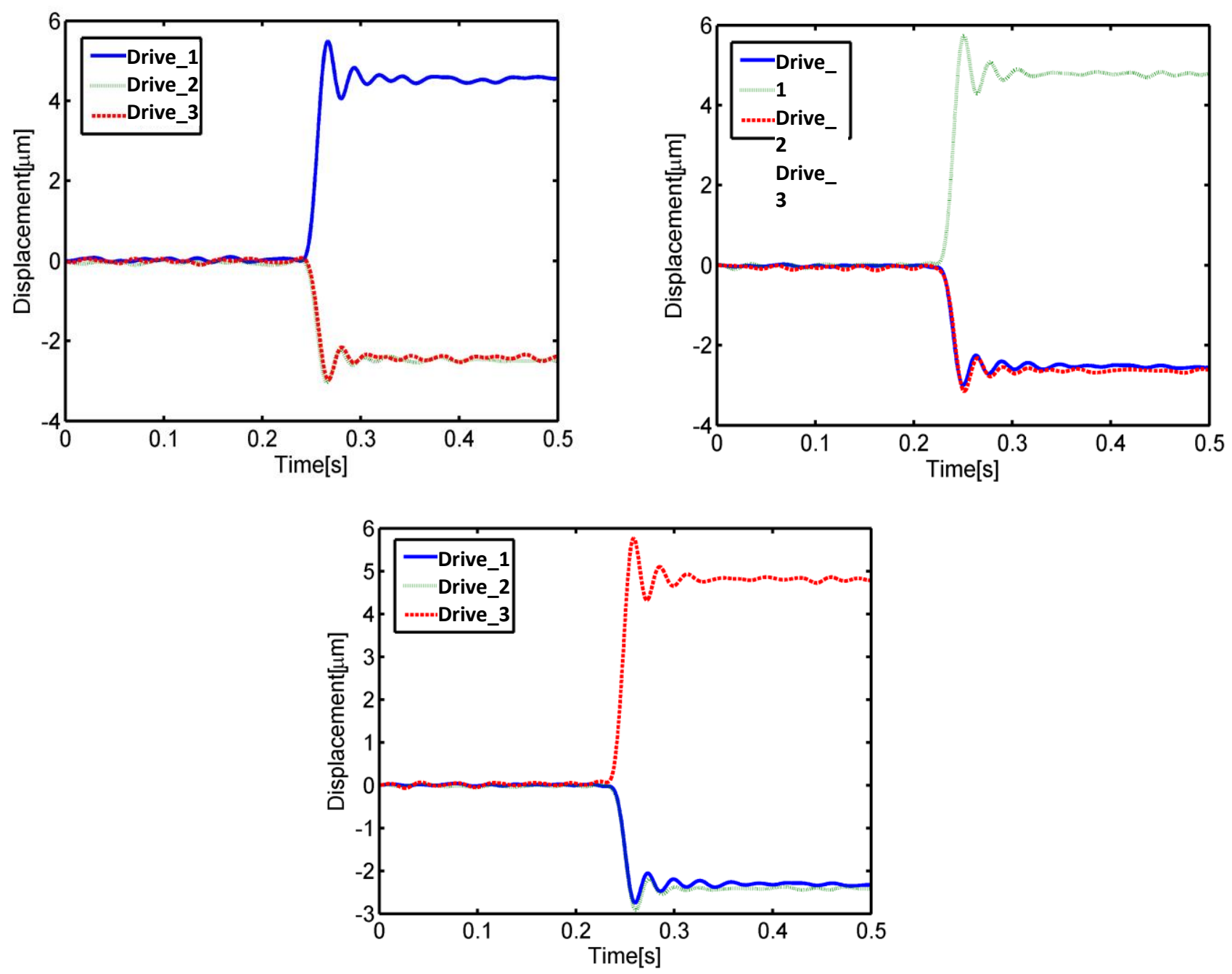

Figure 10. Open-loop step response for each actuation axis. When one axis is actuated, the other two axes are also loaded because of the interaction between the kinematic chains through the end-effector platform.

as the actuation voltage is swept from $-40 \mathrm{~V}$ to $40 \mathrm{~V}$. The voltage and the capacitance provide enough information to determine the electrical energy input into the system. The displacement of the stage, $\Delta Z$, for different actuation voltages is measured by the previously described active probe device [6]. Table 3 lists the total change in capacitance for different driving voltages and the measured displacement $\Delta \mathrm{Z}$. From this data, we calculated values for the total change in energy of the system $\Delta E$, and the corresponding values of $K_{z}$. It can be observed the average value $K_{z}$ is $157.32 \mathrm{~N} / \mathrm{m}$ corresponding to an error with respect to the close-form solution of $5.42 \%$. Among the sources of error are the assumptions of a linearized form of the kinematics in the derivation of the close-form of the stiffness and fabrication imperfections such as over etching of the hinges and springs.

\section{B. Dynamic Characterization}

The time-domain behavior of the device is characterized by using an open-loop step and frequency response analysis. To obtain the step response of the manipulator, an experimental procedure was devised consisting of the following steps:

- Command the end-effector to the position vector $\mathbf{x}=[0,0, \Delta z]^{\mathrm{T}}$ by applying the same bias to all of the actuators. The displacement $\Delta \mathrm{z}$ corresponds to a motion of the end effector close to the half of its total range of motion in the Z-direction.

- Further increment the actuation voltage in one of the actuators by a known value $\Delta \mathrm{V}$ while recording the capacitance changes (i.e., the displacement value) in all of the sensing combs.

- Repeat the previous step for the remaining actuators.

Besides the open-loop characteristics of the device (overshoot, rise time and setting time), this test also verifies its structural integrity. Figures 10(a)-(c) show the change in displacement of the actuators resulting from the step input to one them. As the latter moves forward due to a step in the actuation voltage, the other two are pushed back by approximately half of the displacement of the step. This is expected from the rotational symmetry in XY-plane of the kinematic chains and verifies that the three kinematic chains are mechanically connected through the end-effector. From the time-domain signals the average overshoot, $0 \%-100 \%$ rise 

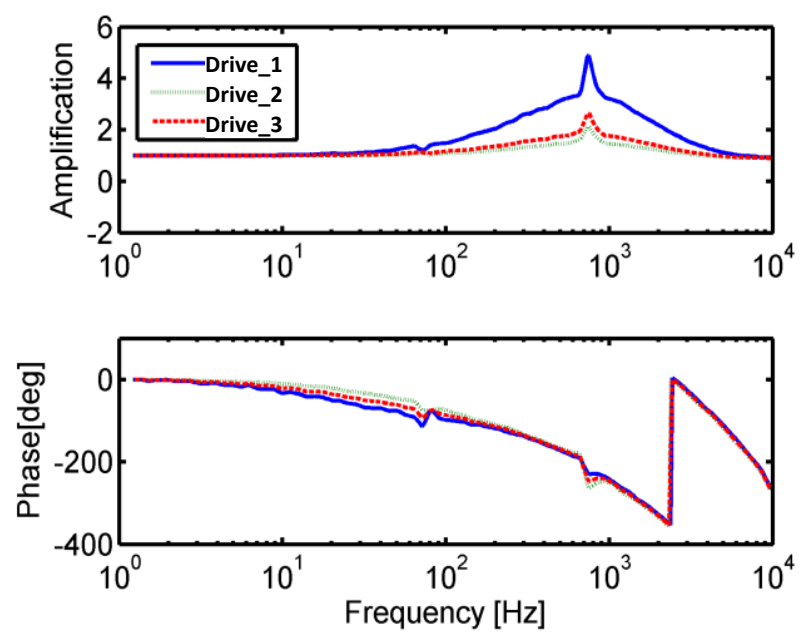

Figure 11. Bode plots of response of actuators of the PK-XYZ MEMS stage

time, and setting times are calculated to be $20.3 \%, 21 \mathrm{~ms}, 91.5 \mathrm{~ms}$ respectively. The overshoot and the settling time observed are typical of flexure-based systems that usually behave as of underdamped systems. They can be modulated by using appropriate feedback control.

To gain further understanding of the dynamics of the system, the bode plot of the open-loop response was generated. Referring to the companion paper [1], the first mode of the structure is in the XY-plane and every direction in the plane is potentially an eigenvector because of in-plane symmetry. In this experiment, three independent bode plots are obtained using dynamic signal analyzer. Each comb actuator is excited with a voltage from a digital signal analyzer (Agilent 35670A), $V=V_{\text {offset }}+$ $V_{\text {excite }} \sin (\omega t)$, where $V_{\text {offset }}$ is much larger (around 40-60V) than $V_{\text {excite }}$ (around 0.05-0.1V) while it's sensing comb's output is recorded by the other channel of the analyzer. The other two are excited with only $V_{\text {of } f s e t}$. Besides characterizing the stage around the center of its workspace, this excitation addresses the parabolic relationship between actuation voltage and the displacement, by making the excitation component containing $\sin ^{2}(\omega t)$ much smaller than that containing $\sin (\omega t)$. The amplification factor and phase information are recorded as the frequency range of $1 \mathrm{~Hz}$ to $10 \mathrm{kHz}$ is scanned. The plot in Figure 11 shows an early peak around $60 \mathrm{~Hz}$, corresponding to line noise, and a second peak at $750.7 \mathrm{~Hz}$ that corresponds to the first mode of vibration of the structure (the in-plane mode). The peak output of the sensing comb corresponding to the first drive comb, and shown as a solid line, is larger and more pronounced than the other two. This may result from the eigenvector of an excited in-plane mode being aligned with the direction of drive 1. From the companion paper [1], an approximate value for the resonant frequency of the structure for this mode is calculated as:

$$
f=\frac{1}{2 \pi} \sqrt{\frac{K}{m}}
$$

$K$ is the predicted stiffness corresponding to the lowest mode of structure. With $K=96.06 \mathrm{~N} / \mathrm{m}$ and a moving mass approximated value of $4.83 \mathrm{e}-6 \mathrm{~kg}$, we get a resonant frequency of $f=709 \mathrm{~Hz}$. The discrepancy between the experimentally observed and predicted values of the first resonance frequency can be explained by the error introduced from assuming a lumped mass at the end-effector, linearized kinematics, imperfect symmetry, and inaccuracies in fabrication.

\section{Conclusions}

In this paper, we have realized a PK-XYZ-MEMS nanopositioning stage based on the parallel kinematics scheme discussed in [1]. We have demonstrated that by using a double device layer SOI substrate, such a system can be fabricated using only robust, high-yield, conventional microfabrication processes and relatively standard MEMS design elements such as electrostatic comb actuators and sensors, folded leaf springs, hinges, flexure parallelogram 4-bar mechanisms and out-of-plain hinges.

The voltage-displacement and displacement-capacitance characteristics were obtained for stage's actuation and sensing combs. These, along with a COTS capacitance-to-voltage conversion chip, a low noise voltage source and a digital signal processor-based embedded control board, were used to construct a rudimentary PID-based closed loop controller for the actuation in each of the stage's three kinematic chains. The closed-loop controllers are meant to facilitate further characterization of the stage by attenuating the effect of forces (especially internal forces generated through interaction of the kinematic chains) on the actuator displacement.

With the help of the closed loop control of the actuators, the kinematics of out-of-plane and in-plane motion were validated. Out-of-plane motion (in the direction of the Z-axis of the stage) of end-effector platform was shown to meet design specifications of $20 \mu \mathrm{m}$. In-plane kinematics of the stage were verified by tracking and measuring the stage's displacements along circles in three different plane. Circular trajectories calculated using inverse kinematics are successfully generated for different height of endeffector with an average error of about $50 \mathrm{~nm}$, most of which might be attributed to the external measurement system. The 
experimental stiffness in the out-of-plane direction was measured using an energy-based methodology and found to be $157.5 \mathrm{~N} / \mathrm{m}$ with error of $5.42 \%$ with respect to analytic solution for stiffness in that direction. The dynamic interactions of the kinematic chains of the device was analyzed in both, the time and frequency domains. Step inputs at different actuation combs produced the desired response in the other chains. The open-loop frequency response of the device indicated resonance at $750 \mathrm{~Hz}$, consistent with the predicted in-plane modal frequency.

The envisioned applications for this stage include mechanical nano-indentation testing, direct writing process, scanning tunneling microscope (STM), and 3D imaging process using micro lens. Future work will first concentrate on developing calibration techniques and synthesizing high-performance closed-loop controllers for position and force control with such MEMS stages.

\section{Acknowledgment}

This work was supported in part by the National Science Foundation under grants CMMI-0749028 and 0800863. Funds from the Tungchao Julia Lu Professorship in the Department of Mechanical Science and Engineering also made this work possible. The authors acknowledge the use of the Micro-Nano Mechanical Systems Laboratory and the Micro-Nano Technology Laboratory for the fabrication and characterization of the devices reported in this paper. Nick Toombs prepared the 3-D renderings of the device.

\section{Reference}

[1] J. Correa, B. Koo and P. Ferreira, "Parallel-kinematics XYZ MEMS Part 1: Kinematics and design for fabrication", Precision Engineering (submitted), 2015.

[2] S. Warnat, H. King, C. Forbrigger and T. Hubbard, "PolyMUMPs MEMS device to measure mechanical stiffness of single cells in aqueous media", Journal of Micromechanics and Microengineering, vol. 25, no. 2, p. 025011, 2015.

[3] B. Koo, X. Zhang, J. Dong, S. Salapaka and P. Ferreira, "A 2 Degree-of-Freedom SOI-MEMS Translation Stage With ClosedLoop Positioning”, J. Microelectromech. Syst., vol. 21, no. 1, pp. 13-22, 2012.

[4] J. Dong and P. Ferreira, "Simultaneous Actuation and Displacement Sensing for Electrostatic Drives," Journal of Micromech. and Microeng., 18, 035011, March 2008.

[5] J. Dong and P. Ferriera, "Electrostatically Actuated Cantilever with SOI-MEMS Parallel Kinematic XY Stage", IEEE-ASME J MEMS, vol. 18, no. 3, pp. 641-651, 2009.

[6] B. Koo and P. Ferreira, "An active MEMS probe for fine position and force measurements", Prec. Eng., vol. 38, no. 4, pp. 738-748, 2014.

[7] D. Mukhopadhyay, J. Dong, E. Pengwang and P. Ferreira, "A SOI-MEMS-based 3-DOF planar parallel-kinematics nanopositioning stage", Sensors and Actuators A: Physical, vol. 147, no. 1, pp. 340-351, 2008.

[8] J. Dong, Q. Yao and P. Ferreira, "A novel parallel-kinematics mechanism for integrated, multi-axis nanopositioning", Prec. Eng., vol. 32, no. 1, pp. 20-33, 2008.

[9] Mathworks Corporation, MA. Registering an Image Using Normalized Cross-Correlation - MATLAB \& Simulink Example, 2015. [Online] Available at: http://www.mathworks.com/help/images/examples/registering-an-image-using-normalizedcross-correlation.html

\section{Appendix A}

A proportional-integral (PI) controller is implemented for each axis of device with the technique in [3], [6]. The performance of the controller is verified by obtaining the closed-loop step and sinusoidal response of the system. For each actuation comb, the controller gains are tuned individually to obtain less than $5 \%$ overshoot and $250 \mathrm{~nm}$ phase lag in the displacement range between $10 \mu \mathrm{m}$ and $20 \mu \mathrm{m}$. Figure A-1 and A-2 shows the step and sinusoidal response for one of the combs.

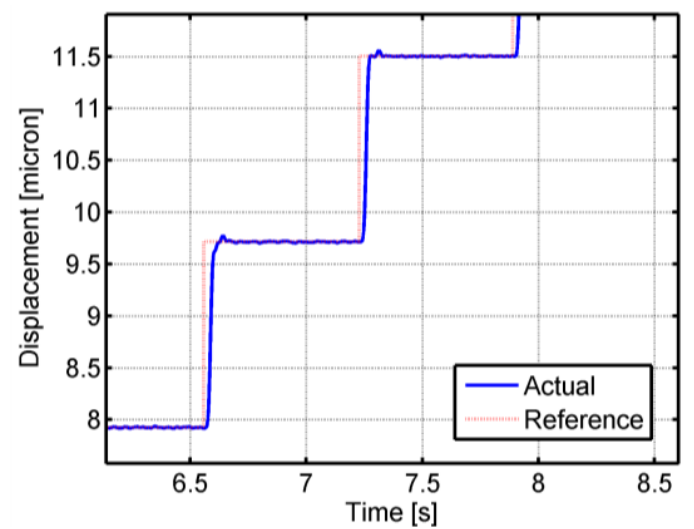

Figure A-1. Closed loop step response of one of the combs of PK-XYZ-MEMS

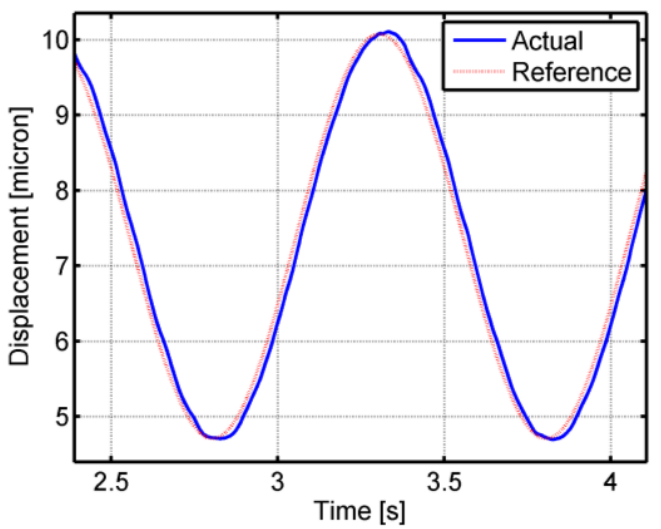

Figure A-2. Closed-loop tracking of a sinusoidal input at one of the combs of PK-XYZ-MEMS 
Graphical Abstract

For

\title{
Parallel-kinematics XYZ MEMS Part 2: Fabrication and Experimental Characterization
}

\author{
Bonjin Koo, Jorge E. Correa and Placid M. Ferreira*
}

Department of Mechanical Science and Engineering, 1206 West Green Street, MC-244, University of Illinois at Urbana-Champaign, Urbana, IL 61801, USA

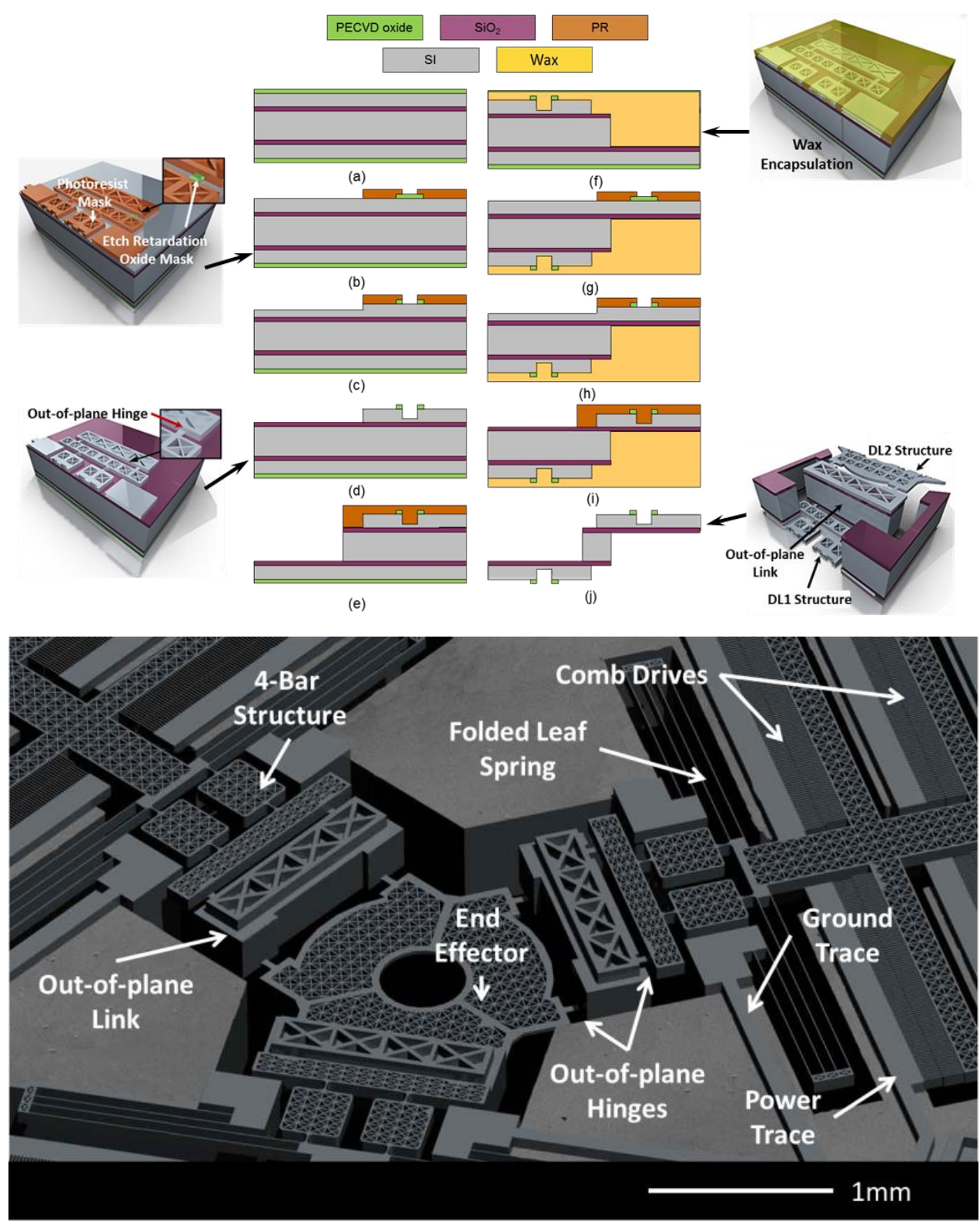

Steps in the process for fabricating a 3-dimensional XYZ MEMS positioning device in a double device layer SOI substrate along with the results of fabrication shown in the electron micrograph 